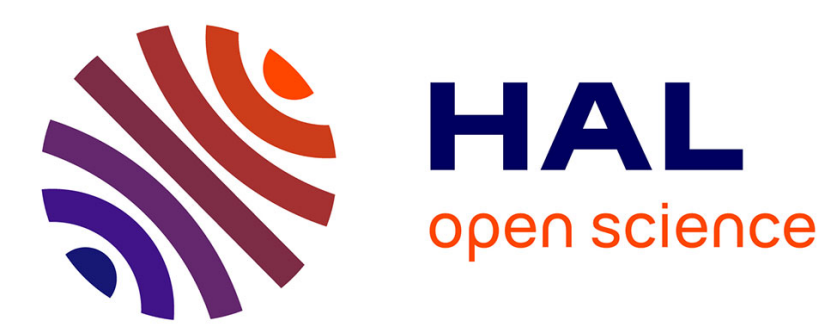

\title{
Diffusion mutuelle de chaînes polymères courtes dans un système de chaînes longues
}

F. Brochard, J. Jouffroy, P. Levinson

\section{To cite this version:}

F. Brochard, J. Jouffroy, P. Levinson. Diffusion mutuelle de chaînes polymères courtes dans un système de chaînes longues. Journal de Physique Lettres, 1983, 44 (12), pp.455-460. 10.1051/jphyslet:019830044012045500 . jpa-00232217

\section{HAL Id: jpa-00232217 https://hal.science/jpa-00232217}

Submitted on 1 Jan 1983

HAL is a multi-disciplinary open access archive for the deposit and dissemination of scientific research documents, whether they are published or not. The documents may come from teaching and research institutions in France or abroad, or from public or private research centers.
L'archive ouverte pluridisciplinaire HAL, est destinée au dépôt et à la diffusion de documents scientifiques de niveau recherche, publiés ou non, émanant des établissements d'enseignement et de recherche français ou étrangers, des laboratoires publics ou privés. 
Classification

Physics Abstracts

$36.20 \mathrm{E}-61.25 \mathrm{H}$

\title{
Diffusion mutuelle de chaînes polymères courtes dans un système de chaînes longues
}

\author{
F. Brochard, J. Jouffroy et P. Levinson \\ Collège de France, Laboratoire de Physique de la Matière Condensée $\left(^{*}\right)$, \\ 11, Place Marcelin-Berthelot, 75231 Paris Cedex 05, France
}

(Reçu le 24 mars 1983, accepté le 2 mai 1983)

Résumé. - Le coefficient de diffusion mutuelle $D$ d'un mélange de deux polymères $A, B$ chimiquement identiques, mais de tailles très différentes $\left(k=\left(N_{\mathrm{B}} / N_{\mathrm{A}}\right) \ll 1\right.$, où $N$ est l'indice de polymérisation) a une forte dépendance en fonction de la composition. Si $\Phi$ est la fraction en volume occupée par les chaînes longues, $D \sim(\Phi / 1-\Phi)$ pour $k \rightarrow 0$. Lorsque l'on met en contact les deux polymères au temps $t=0$, on prévoit alors un profil de diffusion caractérisé par un front très raide du côté des chaînes courtes et plus diffus du côté des chaînes longues. Cette description pourrait peut-être s'appliquer à l'interprétation de certains résultats expérimentaux obtenus par $\mathrm{E}$. Kramer sur les jonctions, $P S_{\left(N_{\mathbf{A}}\right)} / P S_{\left(N_{\mathbf{B}}\right)}$

\begin{abstract}
The mutual diffusion coefficient $D$ of a mixture of two kinds of polymers A and B, which are chemically identical but of greatly different lengths (expressed as $k=\left(N_{\mathrm{B}} / N_{\mathrm{A}}\right) \ll 1$, where $N$ is the degree of polymerization), has a strong composition dependence. In terms of $\Phi$, the volume fraction occupied by the long chains, $D \sim(\Phi / 1-\Phi)$ as $k \rightarrow 0$. Thus, if two polymers are put into contact at $t=0$, the diffusion profile of $\Phi$ is very sharp on the short chain side but very diffuse on the other. This prediction may explain certain experimental results of E. Kramer on polystyrene $P S_{\left(N_{\mathbf{A}}\right)} / P S_{\left(N_{\mathbf{B}}\right)}$
\end{abstract}

\section{Introduction.}

Nous étudions les propriétés dynamiques d'un mélange fondu de deux polymères $\mathrm{A}, \mathrm{B}$ qui sont chimiquement identiques mais qui diffèrent en poids moléculaire (rapport des poids $k=N_{\mathrm{B}} / N_{\mathrm{A}}, N_{\mathrm{A}}$ et $N_{\mathrm{B}}$ étant respectivement les indices de polymérisation des chaînes longues et courtes).

On suppose les chaînes dans le régime "enchevêtré » [1] qui apparaît pour $N_{\mathrm{A}}, N_{\mathrm{B}}>N_{\mathrm{e}}$ $\left(N_{\mathrm{e}} \sim 300\right.$ caractérise la distance chimique entre points d'enchevêtrement) [6].

Pour un tel mélange, l'énergie libre de mélange $F$ est uniquement d'origine entropique. Dans un modèle du type Flory-Huggins, $F$ s'écrit [2] :

$$
\frac{F_{\text {site }}}{k_{\mathrm{B}} T}=\frac{\Phi}{N_{\mathrm{A}}} \log \Phi+\frac{(1-\Phi)}{N_{\mathrm{B}}} \log (1-\dot{\Phi}) .
$$

$\left(^{*}\right)$ Equipe de Recherche Associée au C.N.R.S. (ERA n 542). 
Il faut noter la différence avec le cas des mélanges de polymères $A, B$ compatibles $[7,8]$ où les effets enthalpiques sont essentiels.

\section{Diffusion mutuelle.}

Ecrivons les équations couplées entre les flux $J_{\mathrm{A}}, J_{\mathrm{B}}$ de monomères $\mathrm{A}, \mathrm{B}$ et les forces agissant sur A et B [3] :

$$
\begin{aligned}
& J_{\mathrm{A}}=-\Lambda_{\mathrm{A}} \nabla\left(\mu_{\mathrm{A}}+U\right) \\
& J_{\mathbf{B}}=-\Lambda_{\mathrm{B}} \nabla\left(\mu_{\mathrm{B}}+U\right),
\end{aligned}
$$

$\Lambda_{\mathrm{A}}, \Lambda_{\mathrm{B}}$ sont les coefficients de transfert; $\mu_{\mathrm{A}}, \mu_{\mathrm{B}}$ les potentiels chimiques. $U$ est un potentiel qui assure l'incompressibilité. La condition $\Phi_{\mathrm{A}}+\Phi_{\mathrm{B}}=1$ conduit à $U=-\left(\Lambda_{\mathrm{A}} \mu_{\mathrm{A}}+\Lambda_{\mathrm{B}} \mu_{\mathrm{B}}\right) /\left(\Lambda_{\mathrm{A}}+\Lambda_{\mathrm{B}}\right)$. Remplaçant $U$ par sa valeur dans (2) on trouve :

$$
\mathbf{J}_{\mathrm{A}}=-\Lambda \nabla \mu
$$

où $\mu=\mu_{\mathrm{A}}-\mu_{\mathrm{B}}$ est le « potentiel chimique d'échange » qui se déduit de (1) par $\mu=(\partial F) /(\partial \Phi)$.

$$
\Lambda=\left(\Lambda_{\mathrm{A}} \Lambda_{\mathrm{B}}\right) /\left(\Lambda_{\mathrm{A}}+\Lambda_{\mathrm{B}}\right) \text {. }
$$

Pour des chaînes courtes, les paramètres $\Lambda_{\mathrm{A}, \mathrm{B}}$ sont simplement égaux au produit de la mobilité par monomère $\Lambda_{0}$ par la fraction en volume $\Phi_{\mathrm{A}, \mathrm{B}}$. Dans le cas où les chaînes sont longues [3], le mouvement d'une chaîne est gêné par les contraintes topologiques dues aux autres chaînes. On peut se représenter une chaîne confinée dans un tube de rayon $N_{\mathrm{e}}^{1 / 2} a$ et de longueur $L_{\mathrm{A}, \mathrm{B}}=\left(N_{\mathrm{A}, \mathrm{B}} / N_{\mathrm{e}}\right) N_{\mathrm{e}}^{1 / 2} a$. Pour avancer d'une longueur $l_{\mathrm{A}, \mathrm{B}}=N_{\mathrm{A}, \mathrm{B}}^{1 / 2} a$, la chaîne doit parcourir la distance $L_{A, B}$ beaucoup plus longue. Ceci conduit à une réduction de la mobilité des chaînes d'un facteur $N_{\mathrm{e}} / N_{\mathrm{A}, \mathrm{B}}$. On a donc :

$$
\begin{aligned}
& \Lambda_{\mathrm{A}}=\Phi \Lambda_{0} \frac{N_{\mathrm{e}}}{N_{\mathrm{A}}} \\
& \Lambda_{\mathrm{B}}=(1-\Phi) \Lambda_{0} \frac{N_{\mathrm{e}}}{N_{\mathrm{B}}}
\end{aligned}
$$

L'équation 3 s'écrit alors :

où

$$
\begin{gathered}
\mathbf{J}_{\mathrm{A}}=-D \nabla \Phi \\
D=\frac{\Lambda_{\mathrm{A}} \Lambda_{\mathrm{B}}}{\Lambda_{\mathrm{A}}+\Lambda_{\mathrm{B}}} \frac{\partial \mu}{\partial \Phi} .
\end{gathered}
$$

En utilisant les résultats (4) et (1), on est conduit à un coefficient de diffusion mutuelle :

$$
D=D_{0} \frac{\Phi(1-k)+k}{1-\Phi(1-k)}
$$

avec $k=N_{\mathrm{B}} / N_{\mathrm{A}}$ et $D_{0}=\Lambda_{0} k_{\mathrm{B}} T\left(N_{\mathrm{e}} / N_{\mathrm{A}} N_{\mathrm{B}}\right)$.

Pour $\Phi=0, D=D_{\mathrm{A}}=\Lambda_{0} k_{\mathrm{B}} T / N_{\mathrm{A}}^{2}$. Pour $\Phi=1, D=D_{\mathrm{B}}=\Lambda_{0} k_{\mathrm{B}} T / N_{\mathrm{B}}^{2}$.

Lorsque $k \rightarrow 0, D$ a une forte dépendance en fonction de la composition :

$$
D \sim D_{0} \frac{\Phi}{1-\Phi}
$$




\section{Profil de diffusion. Discussion.}

Dans une expérience en cours de réalisation à Cornell [4], on dépose sur une couche épaisse de polystyrène de grand poids moléculaire $\left(N_{\mathrm{A}} \sim 10^{4}\right)$ des petites billes d'or de taille $100 \AA$, puis une deuxième couche mince (d'épaisseur environ $100 \mu$ ) de polystyrène de masse plus petite $\left(N_{\mathrm{B}} \sim 10^{3}\right)$. On bombarde l'échantillon par des particules $\alpha$. Une mesure sur la perte d'énergie du faisceau réfléchi (proportionnelle à l'épaisseur de la couche mince) permet de suivre le déplacement des billes. Les résultats expérimentaux montrent que la couche de billes reste parfaitement bien localisée et se déplace avec un coefficient de diffusion intermédiaire entre $D_{\mathrm{A}}\left(\sim 1 / N_{\mathrm{A}}^{2}\right)$ et $D_{\mathrm{B}}\left(\sim 1 / N_{\mathrm{B}}^{2}\right)$.

Notre objectif est d'analyser le profil de diffusion attendu lorsque le coefficient de diffusion a la dépendance en concentration décrite par l'équation 6. L'équation de diffusion se déduit de l'équation de conservation en monomère $\mathrm{A}(\partial \Phi / \partial t+\operatorname{div} J=0)$ et de l'équation 5 :

$$
\frac{\partial \Phi}{\partial t}=\frac{\partial}{\partial x}\left[D_{0} \cdot \frac{\Phi(1-k)+k}{1-\Phi(1-k)} \cdot \frac{\partial \Phi}{\partial x}\right]
$$

Le profil est entièrement défini par l'équation 7 et les conditions aux limites

$$
\begin{cases}\Phi=0 & x=\rightarrow-\infty \\ \Phi=1 & x=\rightarrow+\infty\end{cases}
$$

Il est commode de poser $u=x / \sqrt{D_{0} t}$. L'équation 7 s'écrit alors

$$
\begin{gathered}
-\frac{1}{2} u \frac{\mathrm{d} \Phi}{\mathrm{d} u}=\frac{\mathrm{d}}{\mathrm{d} u}\left[\frac{\Phi(1-k)+k}{1-\Phi(1-k)} \frac{\mathrm{d} \Phi}{\mathrm{d} u}\right] \\
\begin{cases}\Phi=0 & u=\rightarrow-\infty, \\
\Phi=1 & u=\rightarrow+\infty .\end{cases}
\end{gathered}
$$

3. 1 Limite $k=0$. L L'équation 9 s'écrit :

$$
-\frac{1}{2} u \frac{\mathrm{d} \Phi}{\mathrm{d} u}=\frac{\mathrm{d}}{\mathrm{d} u}\left[\frac{\Phi}{1-\Phi} \frac{\mathrm{d} \Phi}{\mathrm{d} u}\right] .
$$

Soit $u_{0}$ la valeur de $u$ pour laquelle $\Phi=0$. L'équation 9 montre que

$$
(\mathrm{d} \Phi / \mathrm{d} u)\left(u_{0}\right)=-1 / 2 u_{0} .
$$

Nous avons construit les profils par intégration numérique. On part d'une valeur $u=u_{0}$ pour $\Phi=0$ en choisissant la pente conformément à (11). Les résultats sont représentés sur la figure 1. Pour chaque valeur de $u_{0}$ correspond une valeur limite $\Phi$ quand $u$ tend vers l'infini. La relation $\Phi\left(u_{0}\right)$ est représentée sur la figure 2 . Lorsque $u_{0}$ croît, la longueur de l'intervalle sur lequel $\Phi$ varie de façon significative décroît comme $1 / u_{0}$, on peut donc trouver une solution approchée de $(10)$ en y remplaçant au premier membre - $(u / 2)(\mathrm{d} \Phi / \mathrm{d} u)$ par $-\left(u_{0} / 2\right)(\mathrm{d} \Phi / \mathrm{d} u)$.

Aux grandes valeurs de $u_{0}$, l'équation 10 admet pour solution approchée :

$$
1-\Phi=\mathrm{e}^{-1 / 2\left(\left|u_{0}\right|\left|u-u_{0}\right|\right)} .
$$

Il faut noter que $u_{0}$ diverge pour $\Phi_{\infty} \rightarrow 1$. Ceci nous conduit à étudier en détail le comportement pour $k$ fini. 


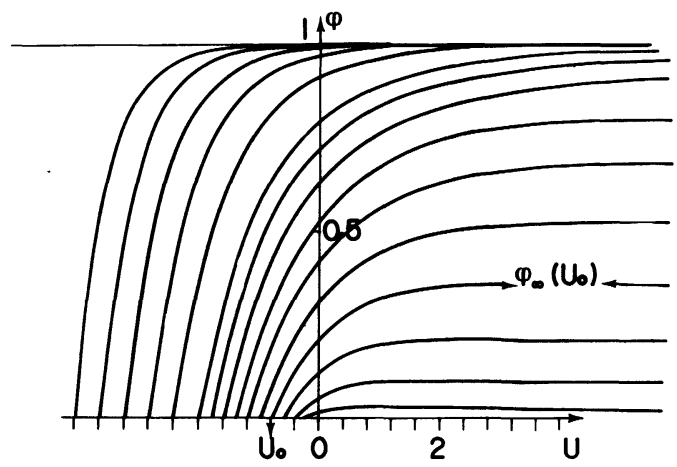

Fig. 1. - Solutions de l'équation : $-1 / 2 u(\mathrm{~d} \tilde{\Phi} / \mathrm{d} u)=(\mathrm{d} / \mathrm{d} u)[(\tilde{\Phi} / 1-\tilde{\Phi})(\mathrm{d} \tilde{\Phi} / \mathrm{d} u)]$ calculées numériquement en variant $u_{0}\left[\Phi\left(u_{0}\right)=0\right]$. La valeur asymptotique $\tilde{\Phi}_{\infty}=\tilde{\Phi}(u \rightarrow \infty)$ en fonction de $u_{0}$ est représentée sur la figure 2 .

[Solution of the equation $-1 / 2 u(\mathrm{~d} \tilde{\Phi} / \mathrm{d} u)=(\mathrm{d} / \mathrm{d} u)[(\tilde{\Phi} / 1-\tilde{\Phi})(\mathrm{d} \tilde{\Phi} / \mathrm{d} u)]$ numerically calculated for various values of $u_{0}\left[\Phi\left(u_{0}\right)=0\right]$. The asymptotic value $\tilde{\Phi}_{\infty}=\tilde{\Phi}(u \rightarrow \infty)$ as a function of $u_{0}$ is shown in figure 2.]

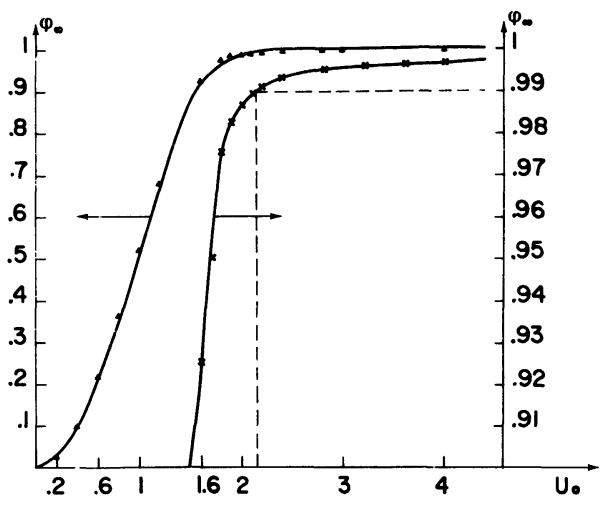

Fig. 2. - Représentation de $\tilde{\Phi}_{\infty}$ en fonction de $u_{0}$. Pour une valeur de $k=N_{\mathrm{B}} / N_{\mathrm{A}}$, on cherche la solution qui correspond à $\tilde{\Phi}_{\infty}=1-k$. Par exemple, pour $k=10^{-2}$, on trouve $u_{0}=2,16$. Pour $k$ strictement nul, $u_{0} \rightarrow-\infty$. On déduit ensuite le profil à partir de la figure 1 .

[ $\tilde{\Phi}_{\infty}$ as a function of $u_{0}$. For a given value of $k=N_{\mathrm{B}} / N_{\mathrm{A}}$, the solution corresponding to $\tilde{\Phi}_{\infty}=1-k$ can be found. For example, for $k=10^{-2}, u_{0}=2.16$ is found. When $k$ is strictly zero, $u_{0} \rightarrow-\infty$. Then, from figure 1 , the profile can be deduced.]

$3.2 k \neq 0(k \ll 1)$. - Pour $k$ petit, mais fini, il est commode de faire le changement de variable :

$$
\tilde{\Phi}=(1-k) \Phi \text {. }
$$

L'équation 9 s'écrit alors

$$
\begin{gathered}
-\frac{1}{2} u \frac{\mathrm{d} \tilde{\Phi}}{\mathrm{d} u}=\frac{\mathrm{d}}{\mathrm{d} u}\left[\frac{\tilde{\Phi}+k}{1-\tilde{\Phi}} \frac{\mathrm{d} \tilde{\Phi}}{\mathrm{d} u}\right] \\
\begin{cases}\tilde{\Phi}=.0 & u=-\infty, \\
\tilde{\Phi}=1-k & u=+\infty .\end{cases}
\end{gathered}
$$


Pour des petites valeurs de $k\left(k \sim 10^{-2}\right)$, on peut négliger $k$ comparé à $\tilde{\Phi}$ dès que $\tilde{\Phi}>k$ et l'on est ramené au cas précédent (Eq. 10), mais pour $u \rightarrow+\infty$, la condition aux limites est $\tilde{\Phi}_{\infty}=1-k$.

Pour $u_{0}$ grand, la solution de (13) s'obtient en remplaçant $\Phi$ par $\tilde{\Phi}$ dans (12) :

$$
1-\tilde{\Phi}=\mathrm{e}^{-1 / 2\left(\left|u_{0}\right|\left|u-u_{0}\right|\right)}
$$

qui montre que $\Phi_{\infty}$ tend vers $1+k$ pour $u_{0} \rightarrow-\infty$.

La solution qui satisfait à $\Phi_{\infty}=1$ correspond donc bien à une valeur de $u_{0}$ fini.

Pour $u_{0}$ quelconque, les solutions de l'équation 13 sont identiques aux solutions de l'équation 10 (Fig. 1). La solution qui satisfait aux bonnes conditions aux limites est celle qui a une valeur limite $\widetilde{\Phi}_{\infty}=1-k ; u_{0}$ se détermine graphiquement à partir de la figure 2 (pour $k=10^{-2}$, on trouve $u_{0}=2,16$ ).

Pratiquement, il est difficile de réaliser des valeurs de $k$ inférieures à $10^{-2}$ (correspondant à $u_{0} \simeq 2$ ). On voit sur la figure 2 que la valeur de $u_{0}$ varie peu pour $10^{-1}<k<10^{-2}$. On a représenté sur la figure 3 la solution correspondant à $k=10^{-2}$. On remarque un front net, avec une pente finie, du côté $\Phi=0\left(\mathrm{~d} \Phi / \mathrm{d} u=-1 / 2 u_{0}=k=10^{-2}\right)$. Par contre, du côté $\Phi=1$, le profil est extrêmement plat.

Dans l'expérience de Kramer, il est plausible de penser que les petites billes pénètrent difficilement dans le réseau des grandes chaînes et donc que le front de billes reste à la frontière du domaine de mélange, défini par $x=-u_{0} \sqrt{D_{0} t}$. L'analyse du déplacement devrait donc donner une détermination de $D_{0}$. Pour avoir tout le profil, il faudrait une autre expérience : par exemple, deutérer l'une des espèces et analyser la composition $\Phi(x)$ par spectroscopie infrarouge [5].

Enfin si l'on suppose que $k$ n'est pas assez petit pour être négligé devant $\Phi$, on ne peut plus supposer $D=0$ pour $\Phi=0$. On a tracé numériquement le profil pour deux valeurs de $k$ (Fig. 3 ). On voit que la propriété essentielle (grande dissymétrie du profil) est maintenue.

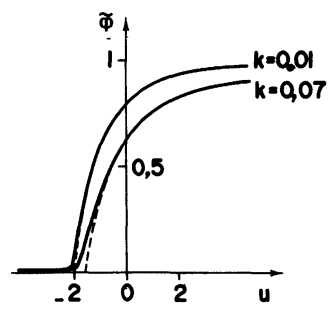

Fig. 3. - Solutions de l'équation : $-1 / 2 u(\mathrm{~d} \tilde{\Phi} / \mathrm{d} u)=(\mathrm{d} / \mathrm{d} u)[(\tilde{\Phi}+k / 1-\tilde{\Phi})(\mathrm{d} \tilde{\Phi} / \mathrm{d} u)]$ calculées pour deux valeurs de $k$. Si $k$ est petit, le profil est bien décrit par l'équation 10 (Fig. 1). Si $k$ devient grand, le front brutal pour $\Phi=0$ s'atténue.

[Solution of the equation, $-1 / 2 u(\mathrm{~d} \tilde{\Phi} / \mathrm{d} u)=(\mathrm{d} / \mathrm{d} u)[(\tilde{\Phi}+k / 1-\tilde{\Phi})(\mathrm{d} \tilde{\Phi} / \mathrm{d} u)]$ calculated for two values of $k$. When $k$ is small, the profile is well described by equation 10 (Fig. 1). As $k$ grows larger, the sharp feature at $\Phi=0$ becomes rounded.]

\section{Conclusion.}

Pour des chaînes polymères $(\mathrm{A}, \mathrm{B})$ de longueurs très différentes mais chimiquement identiques mises en contact à l'instant $t=0$, on prévoit un profil de diffusion à l'instant $t$ :

- très raide du côté des chaînes longues, 
- contrôlé par un coefficient d'interdiffusion donné par la moyenne géométrique des coefficients de diffusion $D_{\mathrm{A}}$ d'une chaîne $\mathrm{A}$ dans un fondu de chaînes $\mathrm{B}$ et $D_{\mathrm{B}}$ d'une chaîne $\mathrm{B}$ dans un fondu de polymères $A\left(D \sim \sqrt{D_{A} D_{B}}\right)$.

Ces résultats montrent aussi que si l'on veut mesurer $D_{\mathrm{A}}$ (ou $D_{\mathrm{B}}$ ) comme dans l'expérience de Klein [5], il faut réaliser une jonction de deux échantillons, l'un de polymère $A$, l'autre de polymère $\mathrm{B}$ contenant une concentration très faible en polymère $\mathrm{A}\left(\Phi \ll k=N_{\mathrm{B}} / N_{\mathrm{A}}\right)$. Si ces conditions ne sont pas réalisées, on mesurera une combinaison des deux. L'analyse des profils de diffusion devrait permettre de déterminer le coefficient de diffusion mutuelle et tester de façon assez détaillée le modèle de reptation pour des mélanges de polymères fondus.

\section{Bibliographie}

[1] Graessley, W., J. Polym. Sc. 92 (1980) 9.

[2] Flory, P. J., Principles of Polymer Chemistry (Ithaca, N.Y., Cornell Univ. Press) 1953.

[3] De Gennes, P. G., J. Chem. Phys. 72 (1980) 4756.

[4] Kramer, E. J., Santa Barbara, Gordon Conference on Polymers (1983). Private communication.

[5] Klein, J., Macromolecules 14 (1981) 460.

[6] Graessley, W., J. Polym. Sc. Polym. Phys. 18 (1980) 27.

[7] Gilmore, P. T., Falabella, R. and Lawrence, R. L., Macromolecules 13 (1980) 880.

[8] Brochard, F., Jouffroy, J. and Levinson, P., Macromolecules (à paraître). 